\title{
RECENT APPROACHES TO ARABIC DIALOGUE ACTS CLASSIFICATIONS
}

\author{
AbdelRahim A. Elmadany ${ }^{1}$, Sherif M. Abdou ${ }^{2}$ and Mervat Gheith ${ }^{1}$ \\ ${ }^{1}$ Institute of Statistical Studies and Research (ISSR), Cairo University \\ ar_elmadany@hotmail.com, mervat_gheith@yahoo.com \\ ${ }^{2}$ Faculty of Computers and Information, Cairo University \\ sh.ma.abdou@gmail.com
}

\begin{abstract}
Building Arabic dialogue systems (Spoken or Written) has gained an increasing interest in the last few. For this reasons, there are more interest for Arabic dialogue acts classification task because it a key player in Arabic language understanding to building this systems. This paper describes the results of the recent approaches of Arabic dialogue acts classifications and covers Arabic dialogue acts corpora, annotation schema, utterance segmentation, and classification tasks.
\end{abstract}

\section{KEYWORDS}

Arabic Dialogue Acts, Spoken Dialogue Acts, Arabic Dialogue Language Understanding.

\section{INTRODUCTION}

Arabic is one of a class of languages where the intended pronunciation of a written word cannot be completely determining by its standard orthographic representation; rather, a set of special diacritics are needs to indicate the intended pronunciation. Different diacritics for the same spelling form produce different words with maybe different meanings. These diacritics, however, are typically omitted in most genres of written Arabic, resulting in widespread ambiguities in pronunciation and (in some cases) meaning. While native speakers are able to disambiguate the intended meaning and pronunciation from the surrounding context with minimal difficulty, automatic processing of Arabic is often hampered by the lack of diacritics. Text-to-speech (TTS), Part-Of-Speech (POS) tagging, Word Sense Disambiguation, and Machine Translation (ML) can be enumerated among a longer list of applications that vitally benefit from automatic discretization (Al-Badrashiny, 2009). Moreover, there are three categories of Arabic language: Classic Arabic "The old written form", Modern Standard Arabic (MSA) "The famous written form today", and dialectal Arabic "Native spoken languages of Arabic speakers" (Diab and Habash, 2007). Since, the written form of the Arabic language - MSA- is differs from dialectal Arabic. However, MSA used primarily for written form but the regional dialects is prevalence in spoken communications or day-to-day dealings. Unlike MSA, the dialects does not have a set of written grammars rules and have different characteristics e.g. morphology, syntax and phonetics. Moreover, Dialectal Arabic can mainly divided into six dialects groups: Maghrebi, Egyptian, Levantine, Gulf, Iraqi and other. Those regional dialects of Arabic are differ quite a bit from each other. Egyptian dialect commonly known as Egyptian colloquial language is the most widely understood Arabic dialect (Zaidan and Callison-Burch, 2012).

David C. Wyld et al. (Eds) : CCSIT, SIPP, AISC, NLP - 2015

pp. 117-129, 2015. (C) CS \& IT-CSCP 2015

DOI : $10.5121 /$ csit.2015.50412 
In this paper, we focus on language understanding component for Arabic dialogues system. However, there are few works have developed for Arabic spoken dialogue system either MSA or dialect as the best of our knowledge; this is mainly due to the lack of tools and resources that are necessary for the development of such systems (Zaghouani, 2014; Lhioui et al., 2013). Therefore, building language-understanding component for dialogue system is requiring four parts: (1) Dialogue Acts Annotation Schema (2) Dialogue corpus (3) Segmentation Classification (4) Dialogue Acts Classification; consequently, this paper present a survey for these parts.

This paper is organized as follows: section 2 present the concepts and terminology that's used in the paper, section 3 present Arabic language understanding components (dialogue acts annotation schema, dialogue corpus, segmentation classification, and dialogue acts classification); and finally the conclusion and feature works are reported in section 4.

\section{CONCEPTS AND TERMINOLOGIES}

This section present the concepts that related to language understanding and used in this paper.

\subsection{Dialogue Act}

The terminology of speech acts has been addressed by Searle (1969) based on Austin work (1962) as (Webb, 2010):

- Assertive commit the speaker to the truth of some proposition (e.g. stating, claiming, reporting, announcing)

- Directives attempts to bring about some effect through the action of the Hearer (e.g. ordering, requesting, demanding, begging)

- Commissures commit speaker to some future action (e.g. promising, offering, swearing to do something)

- Expressive are the expression of some psychological state (e.g. thanking, apologizing, congratulating)

- Declarations are speech acts whose successful performance brings about the correspondence between the propositional content and reality (e.g. resigning, sentencing, dismissing, and christening).

Dialogue act is approximately the equivalent of the speech act of Searle (1969). Dialog acts are different in different dialog systems. So, Major dialogue theories treat dialogue acts (DAs) as a central notion, the conceptual granularity of the dialogue act labels used varies considerably among alternative analyses, depending on the application or domain (Webb and Hardy, 2005). Hence, within the field of computational linguistics - recent work - closely linked to the development and deployment of spoken language dialogue systems, has focused on the some of the more conversational roles such acts can perform. Dialogue act (DA) recognition is an important component of most spoken language systems. A dialog act is a specialized speech act. DAs are different in different dialog systems. The research on DAs has increased since 1999, after spoken dialog systems became commercial reality (Stolcke et al., 2000). So, (Webb, 2010) define the DAs as the labelling task of dialogue utterance that serve in short words a speaker's intention in producing a particular utterance.

\subsection{Turn vs Utterance}

In natural human conversation, turn refer to the speaker talking time and turn-taking refer to the skill of knowing when we start and finish the turn in the conversion. The turn boundary contains 
one or more sentences moreover, the "turn-taking" is generally fixed to the expression of a single sentences. In the spoken dialogue system the term of utterance is refer to the one speech act. (Traum and Heeman, 1997) has defines the utterance unit by one or more of the following factors:

1. Speech by a single speaker, speaking without interruption by speech of the other, constituting a single Turn.

2. Has syntactic and/or semantic completion.

3. Defines a single speech act.

4. Is an intonational phrase.

5. Separated by a pause.

Consequently, this paper refers to an utterance as a small unit of speech that corresponds to a single act (Webb, 2010; Traum and Heeman, 1997). In speech research community, utterance definition is a slightly different; it refers to a complete unit of speech bounded by the speaker's silence while, we refer to the complete unit of speech as a turn. Thus, a single turn can be composed of many utterances. Moreover, turn and utterance can be the same definition when the turn contains one utterance as used in (Graja et al., 2013) . Here an example of a long user turn from Arabic dialogues corpus that contains many utterances (Elmadany et al., 2014):

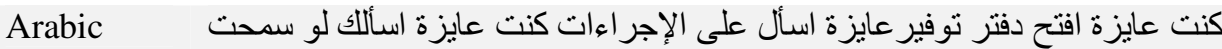

Buckwalter lw smHt knt EAyzp As>lk knt EAyzp AftH dftr twfyr EAyzp As>1 Ely Al<jrA'At

English Excuse me I want to ask you I want open an account I need to know the proceeds

This turn contains four utterances as:

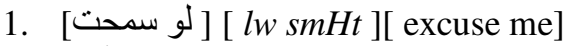

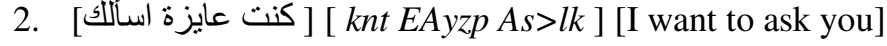

3. [ كنت عايزة افتح دفتر توفيرة [ Ent EAyzp AftH dftr twfyr ] [I want open an account]

4. [عايزة اسأل على الإجر اءات] [EAyzp As>l Ely Al<jrA'At ] [ I need to know the proceeds]

\section{LANGUAge Understanding COMPONENT}

In this section, we present the recent researches for the four parts of building languageunderstanding component for Arabic dialogue systems, these parts are (1) Dialogue Acts Annotation Schema (2) Dialogue corpus (3) Segmentation Classification (4) Dialogue Acts Classification.

\subsection{Dialogue Acts Annotation Schema}

The idea of dialogue act plays a key role in studies of dialogue, especially in communicative behaviour understanding of dialogue participants, in building annotated dialogue corpora and in the design of dialogue management systems for spoken human-computer dialogue. Consequently, to build annotated dialogues corpus we need annotation schema that contains a list of predefined categories, semantic labels, or dialogue acts; schema is considering the key player to build the annotated corpus and dialogue acts classification task.

Searle (1969) has addressed the history of dialogue acts schema (see section 2.1). Moreover, the research on dialogue acts is increasing since 1999 after spoken dialogue systems become a commercial (Stolcke et al., 2000). Many dialogue acts schema applied in non-Arabic dialogues such as English and Germany such as SWITCHBOARD-DAMSL schema. 
As the best of our knowledge, all of the previous dialogue acts annotation schemas applied to mark-up dialogue corpora based on non-Arabic languages such as English, German and Spanish. Moreover, there are few efforts were done to propose dialogue acts annotation schemas for Arabic such as

- So, the first attempt was by (Shala et al., 2010) that proposed dialogue acts schema contains 10 DAs:
- Assertion
○ Declaration
○ Question
- Response to Question
○ Greetings
- Command
- Promise/Denial
- Short Response
○ Expressive Evaluation
○ Indirect
○ Request

- (Dbabis et al., 2012) has been improved (Shala et al., 2010) schema; the reported schema based on multi-dimension " 6 th categories" 13 DAs:

Social Obligation
Management
- Opening
- Closing
- Greeting
- Polite Formula
- Introduce
- Thanking
- Apology
- Regret
Turn Management
- Acknowledgement
- Calm
- Clarify

\begin{tabular}{ll} 
- Clarify & - Opinion \\
- Feedback & - Appreciation \\
- Out of topic & - Disapproval \\
- Non understanding & - Accept \\
signal & - Conclusion \\
- Request & - Partial Accept Reject \\
- Question & - Partial Reject \\
- Order & - Argument \\
- Promise & - Justification \\
- Hope & - Explanation \\
- Wish & - Confirmation \\
- Invocation & $\circ$ Answer \\
- Warning & $\circ$ Statement \\
\hline Argumentation &
\end{tabular}

These schemas have applied to mark-up dialogues corpora based on a general conversion discussion like TV talk-show programs.

- (Graja et al., 2013) reported a words semantic labelling schema to mark-up dialogue utterance word-by-word for inquiry-answer dialogues specially train railway stations; this schema contains about 33 semantic labels for word annotation within five dimensions:

$\begin{array}{ll}\text { O Domain concepts } & \text { - Departure_Cpt } \\ \text { - Train } & \text { - Arrival_Cpt } \\ \text { - Train_Type } & \text { - Price_Cpt } \\ \text { - Departure_hour } & \text { - Class_Cpt } \\ \text { - Arrival_hour } & \text { - Trip_time } \\ \text { - Day } & \text { - Ticket_type } \\ \text { - Origin } & \text { o Requests concepts } \\ \text { - Destination } & \text { - Path_Req } \\ \text { - Fare } & \text { - Hour_Req } \\ \text { - Class } & \text { - Booking_Req } \\ \text { - Ticket_Numbers } & \text { - Price_Req } \\ \text { - Ticket } & \text { - Existence_Req } \\ \text { - Hour_Cpt } & \text { - Trip_timeReq }\end{array}$
- Clarification_Req
- Dialogue concepts
- Rejection
- Acceptance
- Politeness
- Salutation (Begin)
- Salutation (End)
○ Link concepts
- Choice
- Coordination
○ Out of vocabulary
- Out


- Recently, (Elmadany et al., 2014) reported a schema based request and response dimensions for inquiry-answer dialogues such as flights, mobile service operators, and banks; this schema contains DAs:

○ Request Acts

- Taking-Request

- Service-Question

- Confirm-Question

- YesNo-Question

- Choice-Question

- Other-Question

- Turn-Assign

- Response Acts

- Service-Answer
- Other-Answer

- Agree

- Disagree

- Greeting

- Inform

- Thanking

- Apology

- MissUnderstandingSign

- Correct

- Pausing

\author{
- Suggest \\ - Promise \\ - Warning \\ - Offer \\ - Other Acts \\ - Opening \\ - Closing \\ - Self-Introduce
}

\subsection{Arabic Dialogue Acts Corpora}

The use of corpora has been a key player in the recent advance in NLP research. However, the high costs of licensing corpora could be a difficult for many young researchers. Therefore, find freely available corpora is clearly a desirable goal, unfortunately; the freely available corpora are mostly not easily found and the most resources available from language data providers are expenses paid or exclusively reserved for subscribers. As the best of our knowledge, Arabic dialogue segmentation processing is considered hard due to the special nature of the Arabic language and the lake of Arabic dialogues segmentation corpora (Zaghouani, 2014). However, there are many annotated dialogued acts corpora for non-Arabic languages, these are the most annotated corpora used in DAs classifications tasks listed in (Webb, 2010) for non-Arabic languages such as:

- MAPTASK ${ }^{1}$ : consist of 128 English dialogues, containing 150,000 words.

- VERBMOBIL ${ }^{2}$ : consist of 168 English dialogues, containing 3117 utterances. This corpus has annotated with 43 distinct Dialogue Acts.

- SWITCHBOARD ${ }^{3}$ : consist of 1155 telephone conversations, containing 205,000 utterances and 1.4 million words.

- AMITIES ${ }^{4}$ : consist of 1000 English human-human dialogues from GE call centres in the United Kingdom. These dialogues containing 24,000 utterances and a vocabulary size of around 8,000 words.

- $\mathbf{A M I}^{5}$ : Contains 100 hours of meeting.

Unfortunately, to found fully Annotated Arabic dialogue acts corpus is more difficult but there are many of Arabic speech corpora prepared for Automatic Speech Recognition (ASR) research/application. Moreover, most of these corpora are available from the LDC or ELRA members with membership fees e.g. CALLHOME corpus ${ }^{6}$ (Canavan et al., 1997). Therefore, as the best of our knowledge, there are some efforts to building a fully annotated corpus for Arabic dialogues such as:

\footnotetext{
${ }^{1}$ Available at http://www.hcrc.ed.ac.uk/maptask/

${ }^{2}$ Available at http://www.phonetik.uni-muenchen.de/Bas/Bas Korporaeng.html

${ }^{3}$ Available at ftp://ftp.ldc.upenn.edu/pub/ldc/public-data/swb1 -dialogact-annot.tar.gz

${ }^{4}$ Available at http://www.dcs.shef.ac.uk/nlp/amities/

${ }_{6}^{5}$ Available at http://groups.inf.ed.ac.uk/ami/corpus/

${ }^{6}$ Available at https://catalog.1dc.upenn.edu/LDC96S35
} 
- $\mathrm{TuDiCoI}^{7}$ (Tunisian Dialect Corpus Interlocutor): Corpus consists of Railway Information from the National Company of Railway in Tunisia (SNCFT) which a transcribed spoken Arabic dialogues; these dialogues are between the SNCFT staff and clients who request information about the train time, price, booking...etc. Moreover, the initial corpus of TuDiCoI has reported by (Graja et al., 2010) containing 434 transcribed dialogues with 3080 utterances includes 1465 staff utterances and 1615 client utterances. So, TuDiCoI corpus has enriched by (Graja et al., 2013) to contain 1825 transcribed dialogues with 12182 utterances includes 5649 staff utterances and 6533 client utterances. In addition, each dialogue consist of three utterances for clients and three utterances for staff; client turn is composed of average 3.3 words. The low words per clients utterances and dialogues length is due to the words used by clients to request for information about railway services. Moreover, the corpus turns are not segmented into utterances because it is sort and they considered the utterance is equal to the turn. Unfortunately, TuDiCoI are not annotated using DAs schema but it is marked-up by word-by-word schema (see section 3.1).

- (Elmadany et al., 2014) is reported a manually annotated Arabic dialogue acts corpus and manually segmented turns into utterances for Arabic dialogues language understanding tasks. It has contains an 83 Arabic dialogues for inquiries-answers domains which are collected from call-centers. Moreover, this corpus contains two parts:

- Spoken dialogues, which contains 52 phone calls recorded from Egyptian's banks and Egypt Air Company call-centers with an average duration of two hours of talking time after removing ads from recorded calls, and It consists of humanhuman discussions about providing services e.g. Create new bank account, service request, balance check and flight reservation. Moreover, these phone calls have transcribed using Transcriber $\AA^{8}$, a tool that is frequently used for segmenting, labeling and transcribing speech corpora.

- Written 'Chat' dialogues, which contain 31 chat dialogues, collected from mobile network operator's online-support 'KSA Zain, KSA Mobily, and KSA STC'.

Building an annotated DAs corpus need four process recoding (for spoken)/ collecting (for chat) dialogues process, transcription process (for spoken only), segmentation process, and annotation process. Moreover, these processes are expensive.

\subsection{Arabic Dialogue Segmentation}

A segmentation process generally means dividing the long unit into meaningful pieces or small units "non-overlapping units" and it is considering one of the important solutions to solve Natural Language Processing (NLP) problems. Definition of segmentation will differ according to the NLP problem such as:

1. When dividing the text into topics, paragraphs, or sentences, properly named Text Segmentation e.g. (Touir et al., 2008; El-Shayeb et al., 2007).

2. When dividing the sentences into a group of words, properly named Phrase Segmentation.

3. When dividing words into its clitics/affix (prefix, stem, and suffix), properly named tokenization e.g. (Diab et al., 2004).

\footnotetext{
${ }^{7}$ Available at https://sites.google.com/site/anlprg/outils-et-cor pus-realises/TuDiCoIV1.xml?attredirects=0

${ }^{8} \mathrm{http}: / /$ trans.sourceforge.net/en/presentation.php
} 
Build a completely Human-Computer systems and the belief that will happens has long been a favourite subject in research science. So, dialogue language understanding is growing and considering the important issues today for facilitate the process of dialogue acts classification; consequently segment the long dialogue turn into meaningful units namely utterances is increasing. Moreover, Human-Computer Dialogues are divided into different types: Speech Dialogues proper name "Spoken Dialogue" which works in waves and Written Dialogues proper name "Chat" or "Instant Massaging" (IM) which works on text. The waveform in spoken dialogues is usually segment the long input into short pieces based on simple acoustic criteria namely pauses "non-speech intervals", this type of segmentation is namely acoustic segmentation; but it's different when working in text such as chat dialogues, here use a linguistic segmentation. Consequently, to improve the human-computer system need for understand spoken dialogue by extracting the meaning of speaker's utterances, the acoustic segmentation is inadequate in such cases that are needed for further processing based on syntactically and semantically coherent units because it is not reflecting the linguistic structure of utterances(Stolcke and Shriberg, 1996). However, segmentation process is known in dialogues language understanding by many titles such as Utterances Segmentations, Turns Segmentations, and Dialogue Acts Segmentations (see section 2.2);

There are many approaches to understanding both dialogues types (spoken and written) for nonArabic languages e.g. English, Germany, France... etc. (Ang et al., 2005; Ivanovic, 2005; Zimmermann et al., 2005; Ding and Zong, 2003). Moreover, understanding Arabic dialogues have gained an increasing interest in the last few years. To the best of our knowledge; there are few works interested in Arabic dialogue acts classification (see section 3.4); these works have used the user's turn as an utterance without any segmentation e.g. (Shala et al., 2010; Bahou et al., 2008; Graja et al., 2013; Lhioui et al., 2013; Hijjawi et al., 2013; Hijjawi et al., 2014). In addition, there are a few works for the Arabic discourse segmentation such as:

- (Belguith et al., 2005) has proposed a rule-based approach based on 83 rules for Arabic text segmentation which extracted from contextual analysis of the punctuation marks, the coordination conjunctions and a list of particles that are considered as boundaries between sentences.

- (Touir et al., 2008) has proposed a rule-based approach based on sentences connectors without relying on punctuation based on empirical study of 100 Articles, each article have between 450 and 800 words, for analysis to extract the connectors. Consequently, they provided term "Passive" for connector that does not imply any cutting point e.g. "و/and /w" and term "Active" for connector which indicates the beginning or the end of a segment e.g. "لكن /but /lkn". In addition, they concluded that Passive connector has useful only when comes before active. Hence, they are tested the approach on 10 articles, each article have 500 to 700 words.

- (Khalifa et al., 2011) proposed a Machine-Learning approach using SVM based on the connector "g/and/w". Moreover, they reported sixth types of "و/and /w" connector that divided into two classes: (1) "Fasl" for a connector that indicates the beginning of segments, and (2) "Wasl" for connector that does not have any effect on segmentation. In additional, they are built a corpus for newspapers and books which includes 293 instances of the connector "و /and /w" and added diacritization marks manually to the corpus text (training and testing) during the preparation steps. However, these approach very similar to (Touir et al., 2008) when considering the connector "و/and /w". 
- (Keskes et al., 2012) proposed a rule-based approach based on three principals: (1) using punctuation indicators principal only (2) using lexical cues principal only (3) using mixed punctuation indicators and lexical cues. In addition, they used 150 news articles (737 paragraphs, 405332 words) and 250 elementary school textbooks (1095 paragraphs, 29473 words) for built the lexical cues and effective punctuation indicators. Moreover, they concluded two types of punctuation indicators: (1) "strong" that always identify the end or the start of the segments such as the exclamation mark (!), the question mark (?), the colon (:) and the semi-colon (;) (2) "Weak" that don't always identify the begin or the begin of the segment segments such as full-stop (.), the comma (,), quotes, parenthesis, brackets, braces and underscores; They reported the mixed punctuation indicators and lexical cues principal has the best results in textbooks and newspapers.

These approaches are not testing on Arabic dialogues that completely differs for newspapers and books articles; and Arabic spontaneous dialogues is properly dialect Arabic, which is informal text.

\subsection{Recent approaches to Arabic Dialogue Acts Classification}

There are two ways to understand the dialogues language (Webb and Hardy, 2005):

- Shallow understanding: It is simple spotting keywords or having lists of, for example, every location recognized by the system. Several systems are able to decode directly from the acoustic signal into semantic concepts precisely because the speech recognizer already has access to this information.

- Deeper analysis: Using linguistic methods; including part-of-speech tagging, syntactic parsing and verb dependency relationships.

Using Machine Learning (ML) for solving the DA classification problem, researchers have not historically published the split of training and testing data used in their experiments, and in some cases methods to reduce the impact of the variations that can be observed when choosing data for training and testing have not been used (Webb, 2010). Moreover, DAs are practically used in many live dialogue systems such as Airline Travel Information Systems (ATIS) (Seneff et al., 1991), DARPA (Pellom et al., 2001), VERBMOBIL project (Wahlster, 2000), and Amities dialogue system (Hardy et al., 2004). Now, we will describe in brief some of DAs approaches over annotated corpora to recognize dialogue acts:

- Several approaches have proposed for DAs classification and N-gram models can be considering the simplest method of DA prediction; predicting the upcoming, DA based on some limited sequence of previous DAs such as (Hardy et al., 2004; Webb, 2010; Webb and Hardy, 2005; Webb et al., 2005a, 2005b; Nagata and Morimoto, 1994; Niedermair, 1992). Moreover, (Reithinger and Klesen, 1997; Boyer et al., 2010; Stolcke et al., 2000) are used Hidden Markova Model (HMM) with N-gram.

- Samuel et al. (1998) used Transformation-Based Learning (TBL) (Brill, 1995) over a number of utterance features, including utterance length, speaker turn and the dialogue act tags of adjacent utterances.

- (Carberry and Lambert, 1999) used a rule-based model of DA recognition that uses three sources of knowledge, linguistic (including cue phrases), contextual and world knowledge. Moreover, the linguistic knowledge is used primarily to identify if the speaker has some belief in the evidence presented, using prior known cue phrases e.g. BUT, or the use of surfacenegative question forms (Doesn't X require Y?) (Webb, 2010). Also (Prasad and Walker, 
2002) are used a rule based learning method in the DARPA Communicator dialogues. More recently, (Georgila et al., 2009) extended (Prasad and Walker, 2002) work to include manually constructed context rules that cover the user side of the Communicator dialogues

- Bayesian approaches have proven to be effective for DAs classification(Webb, 2010); (Grau et $a l ., 2004)$ used Naïve Bayesian over the WITCHBOARD corpus within a tri-gram language model.

- ( Ji and Bilmes, 2005; Ji and Bilmes, 2006) are investigated the use of dynamic Bayesian networks (DBNs) using graphical models and they reported the best performing set of features is a tri-gram model of the words in the utterances combined with a bi-gram model of DA.

These approaches are tested on non-Arabic dialogues e.g. English, Germany, France... etc. which completely differs for Arabic dialogues. Moreover, understanding Arabic dialogues have gained an increasing interest in the last few years. To the best of our knowledge, there are few works interested in Arabic dialogue acts classification such as:

(Bahou et al., 2008) proposed a method for the semantic representations of utterances of spontaneous Arabic speech based on the frame grammar formalism as show in

— Figure 1 and it's tested on Tunisian national railway queries (1003 queries representing 12321 words) collected using Wizard-of-Oz technology. In addition, this method consists of three major steps: a pre-treatment step that includes the normalization of the utterance and its morphological analysis; a step of semantic analysis that assigns semantic tags to each lexical unit of query; and a frame generation step that identifies and fills the semantic frames of the utterance. They reported $37 \%$ recall, $60.62 \%$ precision and $71.79 \%$ as F-Measure for classification with average execution time for the utterance is $0.279 \mathrm{sec}$.

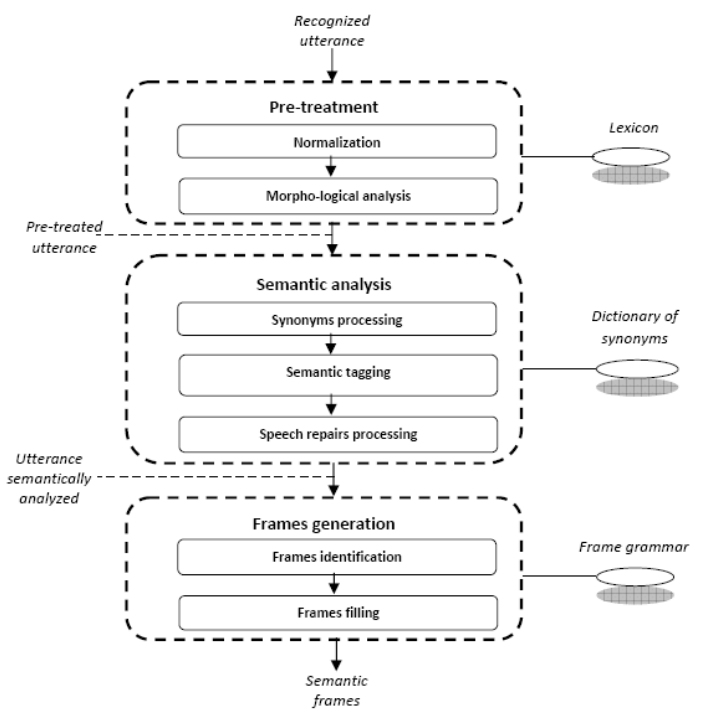

Figure 1. (Bahou et al., 2008) Approach

- (Shala et al., 2010) proposed a fully automated method for speech act classification for Arabic discourse based on the hypothesis that the initial words in a sentence and/or their parts-ofspeech are diagnostic of the particular speech act expressed in the sentence. In addition, used the semantic categorization of these words in terms of named entities and combined this approach with Support Vector Machines (SVM) models to automatically derive the 
parameters of the models they used to implement the approach as show in Figure 2. Moreover, they used two machine-learning algorithms, Naïve Bayes and Decision Trees to induce classifiers acts for Arabic texts and they reported $41.73 \%$ as accuracy scores of all models.

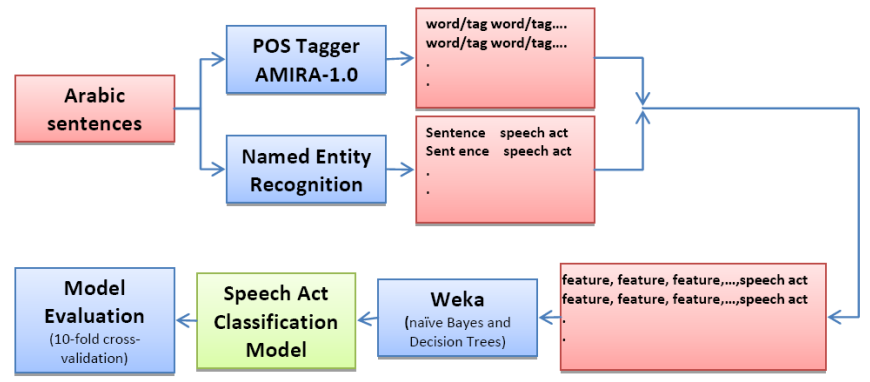

Figure 2. (Shala et al., 2010) Approach

- (Lhioui et al., 2013) proposed an approach based on syntactic parser for the proper treatment of utterances including certain phenomena such as ellipses and it has relies on the use of rule-base (context free grammar augmented with probabilities associated with rules) as show in Figure 3. In addition, they used HHM for creating the stochastic model (if a pretreated and transcribed sequence of words - this words are obviously the output of recognition module - and their annotated corresponding sequences was taken). Moreover, they applied their method on Tunisian touristic domain collected using Wizard-of-Oz technology which contains 140 utterances recorded from 10 speakers with 14 query types (DA) e.g. negation, affirmation, interrogation and acceptance and reported $70 \%$ recall, $71 \%$ precision and $73.79 \%$ as F-measure for classification with average execution time 0.29 seconds to process an utterance of 12 words

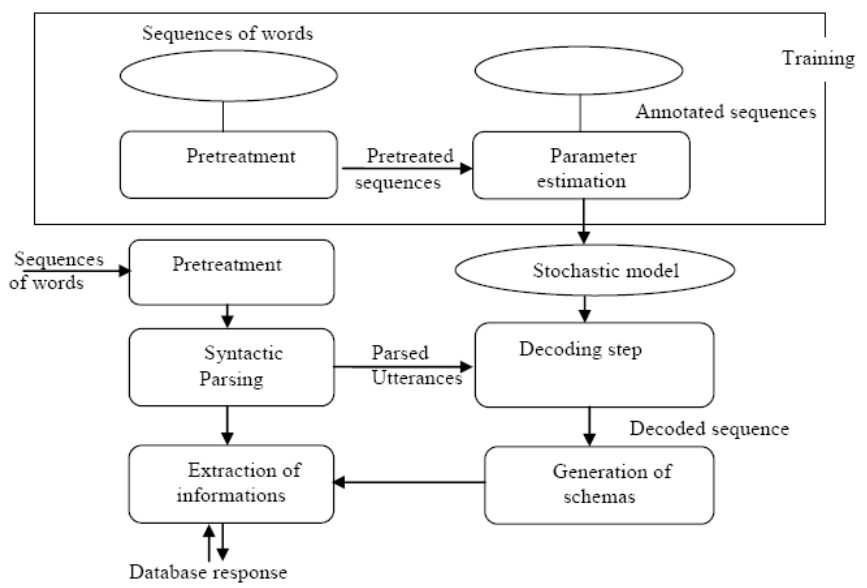

Figure 3. (Lhioui et al., 2013) Approach

- (Graja et al., 2013) proposed discriminative algorithm based on Conditional Random Fields $(\mathrm{CRF})^{9}$ to semantically label spoken Tunisian dialect turns which are not segmented into utterances from TuDiCoI corpus (see section 3.2). Moreover, they applied some treatments to improve turn's structure: (1) lexical normalization such as replacing the word "رزرفسيون"

\footnotetext{
${ }^{9}$ Conditional random fields (CRF) are undirected graphical models trained to maximize a conditional probability which proposed by (Lafferty et al., 2001)
} 
"Reservation” for all its forms e.g. "ريزرفسيون"، "رازارفسيون“, "رازرفسيون“, "رزرفسيون" (2)" Morphological analysis and lemmatization such as replacing the word "خارن" "is going" and " "يخرج" "goes" by the following canonical form " "خرج" "go". (3) Synonyms treatment, this treatment consists in replacing each word by its synonym. In addition, they applied the approach on two data sets one without the treatments and the second with the treatments; and they reported that the treatments has reduce the errors rate compared to the non-treatments data set from $12 \%$ to $11 \%$.

— (Hijjawi et al., 2013) proposed approach based on Arabic function words such as "هل" "do/does", "كيف" "How" and it's focused on classifying questions and non-questions utterances. Moreover, the proposed approach extracts function words features by replacing them with numeric tokens and replacing each content word with a standard numeric token; they used the Decision Tree to extract the classification rules and this approach used on Conversational Agent called ArabChat (Hijjawi et al., 2014) to improve its performance by differentiating among question-based and non-question-based utterances.

\section{Conclusions}

We presented this survey for the recent approaches to Arabic dialogue Acts classification and the goal behind this study is to promote the development and use of Human-Computer research in Arabic dialogues. The results obtained showed that a few works that developed based on Arabic dialogues. Consequently, we hope that this initial attempt to increasing and improve this research as non-Arabic languages.

\section{REFERENCES}

[1] Al-Badrashiny, M. 2009. Autmmatic Diacritizer for Arabic text. MSc in Electronics \& Eletrical Communications. Faculty of Enginnering, Cairo University.

[2] Ang, J., et al. 2005. Automatic Dialog Act Segmentation and Classification in Multiparty Meetings. In Proceedings of IEEE International Conference on Acoustics, Speech, and Signal Processing (ICASSP '05).1061 - 1064.

[3] Bahou, Y., et al. 2008. Towards a Human-Machine Spoken Dialogue in Arabic. In Proceedings of Workshop on HLT \& NLP within the Arabic world: Arabic Language and local languages processing: Status Updates and Prospects, at the 6th Language Resources and Evaluation Conference (LREC'08). Marrakech, Maroc.

[4] Belguith, L., et al. 2005. Segmentation de textes arabes basée sur l'analyse contextuelle des signes de ponctuations et de certaines particules. In Proceedings of 12th Conference on Natural Language Processing (TALN'2005).451-456.

[5] Boyer, K., et al. 2010. Dialogue Act Modleing in a Complex Task-Orinted Domain. In Proceedings of SIGDIAL. Tokyo, Japan:297-305.

[6] Brill, E. 1995. Transformation-based error-driven learning and natural language processing: A case

[7] study in part-of-speech tagging. Computational Linguistics, 21(4):543-565. Canavan, A., et al. 1997. CALLHOME Egyptian Arabic Speech. in L. D. Consortium ed Philadelphia

[8] Carberry, S., and L. Lambert. 1999. A Process Model for Recognizing Communicative Acts and Modeling Negotiation Sub-dialogues. Computational Linguistics.

[9] Dbabis, S. B., et al. 2012. Dialogue Acts Annotation Scheme within Arabic discussion. In Proceedings of SemDial 2012 The 16th workshop on semantics and pragmatics of dialogue Sorbonne, Paris, France.

[10] Diab, M., and N. Habash. 2007. Arabic Dialect Processing Tutorial. In Proceedings of The Human Language Technology Conference of the North American. Rochester

[11] Diab, M., et al. 2004. Automatic Tagging of Arabic Text: From raw text to Base Phrase Chunks. In Proceedings of HLT/NAACL. Boston. 
[12]Ding, L., and C. Zong. 2003. Utterance segmentation using combined approach based on bi-directional $\mathrm{N}$-gram and maximum entropy. Proceedings of the second SIGHAN workshop on Chinese language processing. Association for Computational Linguistics, 17(

[13] El-Shayeb, M., et al. 2007. ArabicSeg: An Arabic News story Segmentation System. In Proceedings of Third International Computer Conference Cairo University (ICENCO 2007). Cairo, Egypt.

[14] Elmadany, A. A., et al. 2014. Arabic Inquiry-Answer Dialogue Acts Annotation Schema. IOSR Journal of Engineering (IOSRJEN), 04(12-V2):32-36.

[15] Georgila, K., et al. 2009. Automatic Annotation of Context and Speech Acts for Dialogue Corpora. Journal of Natural Language Engineering:315-353.

[16] Graja, M., et al. 2010. Lexical study of a spoken corpus in Tunisian dialect.The International Arab Conference on Information Technology (ACIT 2010). Benghazi, Libya.

[17] Graja, M., et al. 2013. Discriminative Framework for Spoken Tunisian Dialect Understanding.SLSP.102-110.

[18] Grau, S., et al. 2004. Dialogue Act Classification using a Bayesian Approach. In Proceedings of 9th Conference Speech and Computer.

[19] Hardy, H., et al. 2004. Data-driven strategies for an automated dialogue system. In Proceedings of the 42nd Annual Meeting on Association for Computational Linguistics.

[20] Hijjawi, M., et al. 2013. User's Utterance Classification Using Machine Learning for Arabic Conversational Agents. In Proceedings of 5th International Conference on Computer Science and Information Technology (CSIT).223-232.

[21] Hijjawi, M., et al. 2014. ArabChat: an Arabic Conversational Agent. In Proceedings of 6th International Conference on Computer Science and Information Technology (CSIT).227-237.

[22] Ivanovic, E. 2005. Automatic utterance segmentation in instant messaging dialogue. In Proceedings of The Australasian Language Technology Workshop.241-249.

[23] Ji, G., and J. Bilmes. 2005. Dialog Act Tagging Using Graphical Models. In Proceedings of IEEE International Conference on Acoustics, Speech, and Signal Processing (ICASSP '05).

[24] Ji, G., and J. Bilmes. 2006. Backo Model Training using Partially Observed Data: Application to Dialog Act Tagging. In Proceedings of the Human Language Technology/ American chapter of the Association for Computational Linguistics (HLT/NAACL'06).

[25] Keskes, I., et al. 2012. Clause-based Discourse Segmentation of Arabic Texts. In Proceedings of The eighth international conference on Language Resources and Evaluation (LREC). Istanbul.

[26] Khalifa, I., et al. 2011. Arabic Discourse Segmentation Based on Rhetorical Methods. International Journal of Electric \& Computer Sciences IJECS-IJENS, 11(01).

[27] Lafferty, J., et al. 2001. Conditional random fields: probabilistic models for segmenting and labeling sequence data. In Proceedings of International Conference on Machine Learning (ICML).282-289.

[28] Lhioui, C., et al. 2013. A Combined Method Based on Stochastic and Linguistic Paradigm for the Understanding of Arabic Spontaneous Utterances. In Proceedings of CICLing 2013, Computational Linguistics and Intelligent Text Processing Lecture Notes in Computer Science. Samos, Greece, 7817(2):549-558.

[29] Nagata, M., and T. Morimoto. 1994. First Steps Towards Statistical Modeling of Dialogue to Predict the Speech Act Type of the Next Utterance. In Proceedings of Speech Communication.

[30] Niedermair, G. 1992. Linguistic Modeling in the Context of Oral Dialogue. In Proceedings of International Conference on Spoken Language Processing (ICSLP'92). Ban, Canada: 63-638.

[31] Pellom, B., et al. 2001. University of Colorado Dialog Systems for Travel and Navigation. In Proceedings of HLT '01: Proceedings of the First International Conference on Human Language Technology Research. USA.

[32] Prasad, R., and M. Walker. 2002. Training a Dialogue Act Tagger for Humna-Human and HumanComputer Travel Dialogues. In Proceedings of the 3rd SIGdial workshop on Discourse and Dialogue. Philadelphia, Pennsylvania.

[33] Reithinger, N., and M. Klesen. 1997. Dialogue Act Classification Using Language Models. In Proceedings of EuroSpeech.

[34] Seneff, S., et al. 1991. Interactive Problem Solving and Dialogue in the ATIS Domain. In Proceedings of HLT '91: Proceedings of the Workshop on Speech and Natural Language. USA:354-359.

[35] Shala, L., et al. 2010. Automatic Speech Act Classification In Arabic. In Proceedings of Subjetividad y Procesos Cognitivos Conference 14(2):284-292.

[36] Stolcke, A., et al. 2000. Dialogue Act Modeling for Automatic Tagging and Recognition of Conversational Speech. Computational Linguistics, 26(3):339-373. 
[37] Stolcke, A., and E. Shriberg. 1996. Automatic linguistic segmentation of conversational speech. In Proceedings of The Fourth International Conference on Spoken Language Processing (LCSLP 96). 2(1005-1008.

[38] Touir, A. A., et al. 2008. Semantic-Based Segmentation of Arabic Texts. Information Technology Journal, 7(7).

[39] Traum, D., and P. A. Heeman. 1997. Utterance units in spoken dialogue. Dialogue processing in spoken language systems Springer. Berlin Heidelberg, 125-140.

[40] Wahlster, W. 2000. Verbmobil: Foundations of Speech-To-Speech Translation Springer.

[41] Webb, N. 2010. Cue-Based Dialogue Act Classification. Ph.D. dissertation. University of Sheffield, England.

[42] Webb, N., and H. Hardy. 2005. Data-Driven Language Understanding for Spoken Language Dialogue American Association for Artificial.

[43] Webb, N., et al. 2005a. Dialogue Act Classification Based on Intra-Utterance Features. In Proceedings of the AAAI Work-shop on Spoken Language Understanding.

[44] Webb, N., et al. 2005b. Empirical Determination of Thresholds for Optimal Dialogue Act Classification. In Proceedings of the Ninth Workshop on the Semantics and Pragmatics of Dialogue.

[45] Zaghouani, W. 2014. Critical Survey of the Freely Available Arabic Corpora. In Proceedings of Workshop on Free/Open-Source Arabic Corpora and Corpora Processing Tools (LREC2014).

[46] Zaidan, O. F., and C. Callison-Burch. 2012. Arabic dialect identification. Computational Linguistics, 52(1).

[47] Zimmermann, M., et al. 2005. Toward Joint Segmentation and Classification of Dialog Acts in Multiparty Meetings. In Proceedings of Proc. Multimodal Interaction and Related Machine Learning Algorithms Workshop (MLMI-05). 